\title{
Musings on Task Shifting in Mental Health
}

\author{
Sharad Philip · Santosh K. Chaturvedi
}

Published online: 21 November 2018

(C) Springer Nature India Private Limited 2018

\section{The Context}

The enormity of the mental health gap has necessitated introduction of innovative methods of care delivery. Qualified and trained resources demand time as the greatest investment amongst other inputs. The projected increase in prevalence of mental illness will further complicate the treatment gap as the burden on specialists and the state infrastructure will only increase. Care services will only be playing a miserable and losing game of catch- up with disease burden and disability. Even in settings with better resource availability, inequitable urbanization and wage discontent have contributed to an exodus of qualified professionals to greener pastures. This has further crystallized the context for task shifting.

\section{The Concept}

To address that resource constraint and consequent health inequity, one of the innovations was to transfer parts of the care process from fewer highly skilled and qualified professionals, to a larger number of not necessarily qualified personnel, specifically trained for those tasks. This process of delegation was recognized

S. Philip · S. K. Chaturvedi ( $₫)$

Psychiatric Rehabilitation Services, Department of Psychiatry, NIMHANS, Bangalore, India

e-mail: skchatur@gmail.com by the World health Organization as task Shifting [1]. Task sharing on the other hand means health professionals working as a team to deliver a service that they may have not carried out before [2]. Capacity building is a process by which institutions and societies set and achieve objectives and become better at solving problems. It is aimed at improving implementation abilities to better respond to perceived needs, available potential, environmental limitations and policy choices. Task shifting has been in use since many decades. The earliest execution of task shifting was the introduction of 'barefoot doctors' in China in the 1920s. They were lay personnel trained for 3 months in carrying out immunization and preventive health programs. However, in the 1980s, this system started losing its popularity due to a variety of reasons, some of which are inadequate training, insufficient incentives and political favoritism. Some health administrators have looked to skills and competency enhancement of existing professionals such as midwives and community nurses [3]. Prescriber training has been quite effective as a force multiplier where there has been a dearth of qualified prescribers. Developed countries such as the USA and the UK have looked to Nurse Practitioners to ensure service delivery in remote areas far from quality care centers.

Health systems reformed to involve nonprofessionals, volunteer and otherwise, as agents to rapidly deescalate the crisis. Specific tasks in these particular instances were shifted from specialists to non-specialists. Tasks such as providing community level basic 
health education, education regarding disease, need for drug compliance, monitoring adherence and side effects etc., were done by non specialists trained for various periods of time. In some situations, competencies of existing health workers were also enhanced so as to be able to transfer less complex tasks from specialist services. This freed up resources to effectively provide specialist care to those who needed it.

\section{The Concept in Mental Healthcare}

The World Health Report 2008 titled 'Primary health Care - now more than ever' emphasized on the urgent need to strengthen primary health care services. Amongst the most significant contexts was the disease burden attributable to mental illness. $45 \%$ of the World's population resides in low resource settings with nil to minimal access to mental health care services [4]. Tasks in mental health care were shifted to psychiatric nurses amongst other para professionals. This has meant better mental health outcomes in such low resource settings. Tasks such as grassroots community education on prevention, surveillance for early recognition, community engagement towards enhanced access and uptake, stigma reduction and better adherence have been performed by non professionals variously, in mental health [5].

\section{Closing the Gap}

In mental health, lay personnel onwards to skilled nurses have been involved in providing accessible care. This has set the stage for attempts at achieving the health related sustainable development goals globally. The motto sums up the effort-leaving no one behind. Tasks such as health surveillance, early identification of illnesses, community sensitization and facilitated adherence have been accomplished through less skilled health workers. The WHO Mental Health Gap Action Program was specifically aimed at reducing the gap in access and uptake of mental health care services. The MHGAP training manual and intervention guide have seen their revision to incorporate modular information resources as well as management algorithms. These are simplified enough to be handy resources for community health workers. These have been tailormade to suit national and subnational settings. But how far are these actually used in practice is not known.

\section{The Concept in Mental Health Rehabilitation}

The concept of recovery is not new to mental illness. Recovery is defined by experts as a way of living a satisfying, hopeful and contributing life despite the limitations caused by mental illness. Rehabilitation is an integral part of mental health recovery. It is the process of enabling persons with disability to obtain and maintain optimal physical, sensory, psychological environmental and social functioning levels. As early as the deinstitutionalization era, mental health professionals and policy makers have seen the need for rehabilitation and reintegration of persons with mental illness, into the society. Community Based Rehabilitation is a more suited and targeted intervention for persons with disability in low- and middle-income countries. The concept of CBR was a major breakthrough by the WHO, in implementing rehabilitation services. In fact, the target audience as mentioned in the WHO CBR booklet includes school teachers, local leaders, primary care workers and even the caregivers of persons with mental illness. Thus, there seems to be simultaneous bridging of the treatment and knowledge gaps.

The different domains of Community Based Rehabilitation including health, education, empowerment, livelihood and social needs can all be addressed by shifting tasks to existing resources. Several examples can be quoted. If we consider treatment adherence, a number of people including employers, teachers and lay counsellors can function as compliance enhancers. The above persons can also be educated for picking up early warning signs of relapse. They can assist in referring patients appropriately. Some aspects of the non pharmacological management of mental ailments can also be delivered by non specialist but trained personnel. For example, like how inclusive education for children with special needs is carried out by non specialist teachers trained by resource teachers, similarly, the same teachers can also be trained in assessing social competence and offering components of social skills training like maintaining interpersonal distance and voice modulation. School mental health programs targeting life skills, resilience promotion, education regarding substance use can be provided by 
ex patients. In a similar manner, employers of PMIs can also function as employment specialists offering opinions on vocational potential and pre vocational skills. Trained staff can also help with prevocational training, helping prepare $\mathrm{CVs}$, teach job interview skills, soft skills, etc., In resource strapped settings with unmet needs and dearth of professionals, even occupational therapy can be task shifted to non professional, but trained staff. Likewise, another resource of health care which can be put to effective use, would be the nursing cadre. The concept of nurse practitioners is already in vogue in some Western countries. Nurse practitioners can also be trained in offering other interventions like cognitive retraining, social skills training, etc., Family counselling and psychoeducation sessions can be done by community members, ex-patients for groups within the community. This will aid in demystifying mental illness and reducing stigma. Hyperlocal onsite interventions in training for ADLs and IADLs for carers of persons with developmental disability can also be shifted to lay health workers. Training of these personnel in basic teaching techniques like shaping, chaining and differential reinforcement can be undertaken.

The crux of a multidisciplinary mental health rehabilitation team would be not the number and variety of professionals from various disciplines but the number of professionals who can task shift amongst each other's disciplines.

\section{Closing the Rehabilitation Gap}

The MHGAP interventions are aimed towards reducing the treatment gap. Current knowledge and practice inform us that adequate treatments seldom result in functional restoration. A significant contributor towards disability/dysfunction would be the impairment due to the mental illness. The corollary of such an orientation would be that there also exists a rehabilitation gap. Thus, now is an appropriate time to introduce a rehabilitation gap intervention guide and training manual that would enable scaling up of rehabilitation services.

\section{The Concerns}

This progress has not been without reservations and apprehensions. From the perspective of service provision, there is a query on whether creating such a cadre to step in and provide interventions will lead to fragmentation and a piecemeal approach to service users. With the vigorous emphasis on individual autonomy and informed choice, would service users choose services provided by nonprofessionals? Would the training be sufficient to empower the trainee to be recognized as a skilled worker while providing the interventions? A task shifting or sharing approach would not be in the interest of holistic management for the service user intuitively.

\section{Ethical Concerns}

The guidelines from the WHO regarding task shifting delineate basic practice standards and ethical principles. However, there is no body or association that will regulate this task force. There is no clear delineated code of conduct. Ethical concerns of utilizing a nonspecialist task force to deliver mental health care services have not been researched. Research literature on task shifting in mental health majorly focusses on examining their effectiveness, impact on access and uptake of services etc.

From the policy makers' points of view, the cost effectiveness makes it lucrative. It would work best as a stopgap solution between the current situation and a better future, when there would be adequate resources-human and otherwise. That would have been a reasonable context to justify introduction of nonprofessionals into professional mental health care services. There follows then a need to simultaneously focus on training more specialists and enhance infrastructure. In such a situation then what would be the role of these community health volunteers and workers? Such a task force is quite prone to unfair employment practices and exploitation at the hands of the program managers. Such volunteers and workers are also not involved in decision making processes or planning of interventions. This would result in disempowering the foot soldiers battling the crisis and reducing the respect for them in the very community they are servicing. Lay health workers or volunteers would be the health system's primary contact. Thus, 
they would be the resource most susceptible to professional carer burnouts. They would also be exposed to traumatic content, stigma, discrimination and human rights violations. Training for shorter periods of time may not equip these nonprofessionals to be able to effectively deal with emotionally charged conversations. Without continued supervision, their own mental health is in jeopardy. This would be a grave travesty of justice. Emphasis on essential care practices such as confidentiality, respect for autonomy, justice, beneficence and/or non-maleficence during training may be insufficient and may require extended periods of supervision. This may not always be possible in the resource strapped settings where task shifting was required in the first place. They also would be at a disadvantage relative to health care team members with regard to career progression in the health services field. Most such community workers also work in a volunteering fashion or are paid far lower than their professional peers. This would engender dissatisfaction and discontent and may even precipitate an exodus.

\section{Concerns with Training}

The training manuals and intervention guides, though comprehensive and designed to only offer evidencebased care in the most simplified terms, still demands a lot of initiative from the trainers and instructors. These training sessions are best conducted in groups and selfreading of the training manuals or the intervention guide may not equip the prospective uninitiated volunteer. There may be a requirement for some extra reading material in their vernacular, that can sufficiently address their curiosity which should be curated by local experts and modified accordingly. Another facet of professional training is the supervised practice and learning by doing. Stipulating certain number of hours of observational training may not be adequate to imbibe interview and psychotherapeutic skills. These programs also do not stipulate minimum qualification ostensibly to attract larger number of volunteers and workers.

In group training, individuals may have different learning curves and grasping potentials. Trainers then would have to be mindful of these concerns as well, while undertaking sessions. Career progression opportunities are also needed to incentivize retention in the community health work.

\section{Legal Concerns}

Another concern is from the legal perspective on task shifting. Here is a cadre of nonprofessionals who have been tasked to provide some aspects of health care. In case of any adverse events arising in this context, how would liability be adjudged? Would it lie solely on the trainer/liaising organization? Would the principle of vicarious responsibility be applicable here? There is neither any research nor a consensus on this facet of task shifting. Regulatory agencies such as the RCI, MCI, INC do not permit nonprofessionals to provide such services. Being the regulatory body and a large body of professionals, their clout is something to be reckoned with. The RCI specifically warns regarding nonprofessionals practicing in the field of rehabilitation.

\section{Systemic Concerns}

Community health programs have so far gone forward in a fragmented approach. Political will plays its part in the identification of which health issue is to be tackled. The picture for individual programs appears strategically sound with the greatest number of staff at the grass roots level. However, when viewing it from the community perspective, the same grassroots workers seem to be the common pivot for all public health programs. From the professional standpoint, there is a concern regarding the dilution of care standards and fragmentation of services. This would also lead to poor integration of health services and thus perpetuate the health inequity which it was supposed to address.

Within such a task force, it is imperative to include persons with lived experience who would have an epistemological advantage. This would greatly enrich the care provided, even though it may not focus on alleviation of symptoms. There would be a definite benefit in terms of exchange of coping strategies, reduction of stigma-internal and external. Their presence can also have a multiplier effect on the training of other members. It would also be a source of encouragement and solace for the beleaguered family 
members of the service users if they were to know regarding the community worker's struggle with mental illness. It is also fraught with confidentiality and privacy concerns. Self revelation should be left to the discretion of the ex-patients. These concerns cannot be taken lightly and may warrant deliberations and negotiations with all the stakeholders.

\section{Continuing Ahead}

In the light of these concerns, it would be prudent to initiate this dialogue sooner rather than later. Now is a better time than ever as efforts to reduce the rehabilitation gap can be administered concurrently while attempting to address the treatment gap, especially in the mental health context. Some of the musings on how the situation can be improved are discussed here. By no means are they comprehensive nor are they based on anything other than the editor's opinion. At the national level, there has to be recognition of the contribution of lay community health workers. There ought to be a national level policy detailing these and other concerns and appropriately addressing them.

Setting minimum thresholds for being able to work in mental health practice is counterproductive in some ways. It may be an obstacle for task shifting as people with requisite qualification may not be readily available. Measures like allowing for apprenticeship or other ways of valuing community work experience in health sector should be introduced. If not involved in direct patient care, Community and Volunteer health workers should at least be involved in curating programs, plans or implementation strategies. They can also be involved in training and skilling future professionals in nuances of cultural behavior, beliefs and explanatory models of illness.

Incentivization strategies should look at composite target and not disaggregated ones from different health programs. Monetary incentives are a good reinforcement strategy but they should not be read as wages for work.
It is imperative to create surveillance programs/ mechanisms to identify service provider burnout and distress. Periodic team building programs, stress relieving time off from their work can achieve these objectives. One other method would be to allow for these cadre to meet together to discuss common emotional health issues amongst themselves in a group. These meetings can be guided discussions keeping in mind the confidentiality and privacy concerns. Periodic supervision and opportunities for case discussions would also serve to build cadre confidence and self competence. Periodic feedback from supervisors at all levels would enable course correction from time to time. They must also be safeguarded against unfair employment practices. A greater emphasis on task shifting is welcome, but the cadre needs to be valued. This force measure would be the appropriate bridge over the mental health and rehabilitation gap.

\section{References}

1. World Health Organization. WHO I First global conference on task shifting. WHO. World Health Organization; 2010. http://www.who.int/mediacentre/events/meetings/task_ shifting/en/. Cited 7 Oct 2018.

2. Dawson AJ, Buchan J, Duffield C, Homer CSE, Wijewardena $\mathrm{K}$. Task shifting and sharing in maternal and reproductive health in low-income countries: a narrative synthesis of current evidence. Health Policy Plan. 2014;29(3):396-408. https://doi.org/10.1093/heapol/czt026.

3. Recommendations W. Optimizing health worker roles to improve access to key maternal and newborn health interventions through task shifting. Geneva: World Heal Organization; 2012.

4. Gauld R, Blank R, Burgers J, Cohen AB, Dobrow MK, Ikegami NKI, et al. The World Health report 2008: primary healthcare: how wide is the gap between its agenda and implementation in 12 high-income health systems? Healthc Policy. 2012;7(3):38-58.

5. For U. mhGAP forum 2009. Ment Health Pract. 2009;88(410):1-31. 